\title{
Delta Neutrophil Index as a Diagnostic Marker of Neonatal Sepsis
}

\author{
Nuriye Asli Melekoglu10 Seyma Yasar $^{2}$ Mehmet Keskin ${ }^{3}$ \\ ${ }^{1}$ Department of Pediatrics, Neonatal Intensive Care Unit, School of \\ Medicine, Malatya Turgut Özal University, Malatya, Turkey \\ 2 Department of Biostatistics and Medical Informatics, Faculty of \\ Medicine, University of Inonu, Malatya, Turkey \\ ${ }^{3}$ Department of Pediatrics, Division of Pediatric Endocrinology and \\ Metabolism, Faculty of Medicine, The University of Gaziantep, \\ Sehitkamil/Gaziantep, Turkey \\ J Pediatr Infect Dis 2021;16:99-105. \\ Address for correspondence Nuriye Asli Melekoglu, MD, Department \\ of Pediatrics, Neonatal Intensive Care Unit, School of Medicine, \\ Malatya Turgut Ozal University, Malatya, Turkey \\ (e-mail: draslicocuk@yahoo.com).
}

\begin{abstract}
Keywords

Objective Sepsis diagnosis is challenging due to nonspecific symptomatology in newborns. Timely diagnosis is essential for reducing sepsis-related morbidity and mortality. This study was performed to determine the diagnostic value of the delta neutrophil index (DNI) for detection of neonatal sepsis and to compare its efficacy with other conventional markers.

Methods This study was conducted at a tertiary hospital in newborns with confirmed sepsis $(n=59)$, suspected sepsis $(n=46)$, and in age- and weight-matched controls $(n=49)$. DNI, white blood cell count, C-reactive protein (CRP) level, and platelet measurements were determined, and blood cultures were performed at the onset of symptoms.

Results The mean DNI was significantly higher in confirmed and clinical sepsis groups compared with the control group. $(6.9 \pm 9.3,1.9 \pm 2.1$, and $0.4 \pm 0.5$, respectively, $p<0.001)$. ROC curve analysis also showed that the combination of DNI and CRP had the highest sensitivity $(86 \%)$, specificity $(100 \%)$, and positive predictive value $(100 \%)$ for predicting neonatal sepsis. DNI values were significantly higher in nonsurvivors $(p<0.05)$.

- delta neutrophil index

- sepsis

- newborn

Conclusion DNI could be used as a reliable diagnostic marker for neonatal sepsis, and high DNI could predict sepsis development and unfavorable outcomes. The diagnostic capability of DNI may be increased by assessing CRP measurements simultaneously.
\end{abstract}

\section{Introduction}

Sepsis continues to be one of the major contributors to morbidity and mortality in the neonatal period, and the lifesaving step is prompt diagnosis and accurate treatment. Although subtle and nonspecific clinical signs make early diagnosis difficult in neonates, timely diagnosis of sepsis is essential to reduce mortality. ${ }^{1}$ Early onset of sepsis (EOS) occurs in the first 3 days of life and it is acquired by vertically. Late onset sepsis (LOS) occurs after postnatal day 3 , and it is

received

August 6, 2020

accepted after revision

December 19, 2020

published online

February 25, 2021 related to vertical transmission or more often hospital-community origin. The gold standard for diagnosis of sepsis is a positive blood/urine/cerebrospinal fluid culture, but this has limited value due to the long duration requirement for any growth and identification of microorganisms and their antibiotic susceptibility (at least 48-72 hours) and low rate of reproduction. Moreover, due to factors such as antenatal use of antibiotics and small blood sample volume, the absence of positive blood culture cannot exclude a diagnosis of sepsis in neonates. ${ }^{2}$ In the current context, the utility of various (c) 2021. Thieme. All rights reserved. Georg Thieme Verlag KG, Rüdigerstraße 14, 70469 Stuttgart, Germany
DOI https://doi.org/ 10.1055/s-0041-1726118. ISSN 1305-7707. 
inflammatory biomarkers, such as acute phase proteins, cytokines, chemokines, and cell adhesion molecules, have been assessed in several studies, to diagnose sepsis earlier than blood culture positivity, but almost none of them have been adopted in general clinical use despite having highnegative predictive values. ${ }^{3}$ Thus, there is still a need for a diagnostic tool that can be used in clinical settings because most of these markers are not readily available for utilization by all health professionals.

Previous studies have focused on the use of hematological parameters to increase the diagnostic yield for neonatal sepsis, especially with the introduction of new parameters, such as white blood cell (WBC) indices and percentage of immature granulocytes (IGs), which are provided by automated hematology analyzers. ${ }^{4,5}$ The granulocytic left shift is characterized by the increase in IGs in the peripheral blood and reflects active bone marrow response to bacterial infection. In addition to being time consuming, the detection of IGs by microscopic count requires experienced staff, resulting in reader bias and limited reproducibility. ${ }^{6}$ Hence, several authors have suggested that manual counting of IGs is an inappropriate method, so its diagnostic value remains controversial in clinical practice. ${ }^{7}$ Modern automated hematology analyzers can measure IG count and percentage in peripheral blood samples. Delta neutrophil index (DNI) is a hematological inflammatory marker that reflects the fraction of IGs in the circulating blood. While several studies ${ }^{8,9}$ have demonstrated a relationship between increased levels of DNI and inflammation/infection in adults and children, only a few studies have assessed the relationship between DNI level and sepsis in neonates. ${ }^{10,11} \mathrm{We}$ hypothesized that there may be a marked increase in DNI in response to bacterial infection in neonates, similar to that seen in adults. Therefore, we investigated the utility of DNI as a diagnostic marker for neonatal sepsis and compared its efficacy with other conventional infection markers, such as WBC, C-reactive protein (CRP), and platelet (PLT) count.

\section{Materials and Methods}

This retrospective study was conducted at the neonatal intensive care unit of Gaziantep University School of Medicine and approved by the institutional research ethics committee (approval number: 2016/302). Sepsis evaluation was based on the clinical symptoms suggestive of infection, such as hyperthermia or hypothermia, apnea, bradycardia, tachycardia, tachypnea, cyanosis, respiratory distress, vomiting, abdominal distension, poor perfusion, hypoactivity, lethargy, hypotonia, and convulsions. Sepsis scanning included complete blood cell count $(\mathrm{CBC})$, peripheral blood smear, CRP, and blood culture. In addition to clinical symptoms if two or more following laboratory abnormalities were present, the patient was diagnosed with sepsis: (1) WBC count of $<5,000$ or $>20,000 / \mathrm{mm}^{3}$; (2) absolute neutrophil count (ANC) of $<1,000$ or $>17,000 / \mathrm{mm}^{3}$; (3) PLT count $<100,000 / \mathrm{mm}^{3}$; (4) immature/total neutrophil ratio of $>0.2$; and (5) CRP measurement $>10 \mathrm{mg} / \mathrm{L}$. The patients with positive blood cultures were considered as "confirmed sepsis" and those with negative blood cultures were considered as "clinical sepsis." The confirmed and clinical sepsis groups were compared with a gestational age- and birth weightmatched control group, which included predischarge nonseptic neonates, hospitalized at the same time for various conditions, such as transient tachypnea of the newborn, jaundice, dehydration, and others. Patients with perinatal asphyxia, patients undergoing surgery, and patients with positive blood cultures for organisms considered to be contaminants were excluded from the study. Positive blood cultures with previously isolated organisms within the 28 days of sepsis onset were considered part of the first bacteremic episode. In the confirmed and clinical sepsis groups, subgroup analysis was performed for survivors and nonsurvivors to evaluate mortality associated with sepsis.

We collected demographic data, including sex, birth weight, gestational age, intrauterine growth restriction, mode of delivery, exposure to antenatal steroids, Apgar's scores at 1 and 5 minutes, history of chorioamnionitis and prolonged rupture of membranes ( $>18$ hours), maternal intrapartum antibiotic usage, and presence of early/late onset of sepsis. As part of the sepsis evaluation, blood samples were drawn for $\mathrm{CBC}$, blood culture, and CRP on the day of clinical suspicion and before the empiric antibiotic treatment. Prophylactic antibiotics were used neonates born to mothers with chorioamnionitis, fever or premature ruptures of membranes ( $>18$ hours) (PROM), and treatment was discontinued when the sepsis diagnosis ruled out after 48 hours later. So, among the patients who were evaluated for risk factors, only those conditions compatible with sepsis were included the study and received antibiotic treatment for at least 10 days depending on their clinical condition or type of microorganism. Complete blood cell count and DNI were measured by using an automated hematology analyzer (ADVIA 2120; Siemens Healthcare Diagnostics, Forchheim, Germany). DNI was calculated by using the following formula: DNI $(\%)=$ (leukocyte subfraction assayed in the myeloperoxidase channel by cytochemical reaction) (leukocyte subfraction counted in the nuclear lobularity channel by the reflected light beam). CRP was determined by the immunoturbidimetric method. A volume of at least $1 \mathrm{~mL}$ of blood was taken by using the aseptic technique and added to pediatric sample-sized blood culture bottles, and then analyzed by using a fully automated BACTEC method (BACTEC 9240 device; Becton Dickinson, Heidelberg, Germany).

The statistical software package IBM SPSS 25.0 (SPSS, Inc.; Armonk, New York, United States) was used for all data analyses. Data are provided as means \pm standard deviation or median ( $\min$ - max) for continuous variables and as numbers or percentages for categorical variables. Normality of the data distribution was checked by using the Kolmogorov-Smirnov test. The independent samples t-test, Pearson's Chi-square test, Fisher's exact test, and Kruskal-Wallis test were used for statistical analysis as appropriate. To assess the diagnostic performance of DNI and other laboratory parameters, ROC curves were constructed and areas under the ROC curves were calculated. Logistic regression analysis was used for odds ratio estimations. Post hoc power analysis was performed on eligible 49 controls, when type 1 error amount was 0.05 and 
the effect size was 0.5 , the power of the study obtained was calculated as 0.99 . In all analyses, $p<0.05$ was taken to indicate statistical significance.

\section{Results}

A total of 526 neonates admitted to the neonatal intensive care unit (NICU) between January 2015 and December 2016, and 412 of them were evaluated for neonatal sepsis. The following patients were excluded from the study: 42 patients undergoing surgery, 16 patients with contaminant blood cultures, and 8 patients with asphyxia. In 241 patients, sepsis diagnosis was ruled out. Therefore, this study was performed in a total of 154 patients, consisting of 59 in the confirmed sepsis group, 46 in the clinical sepsis group, and 49 in the control group. There were no significant differences between the groups in gestational age, birth weight, sex, mode of delivery, Apgar's score at 1 minute, frequency of intrauterine growth restriction, exposure to antenatal steroids, maternal intrapartum antibiotic usage, history of chorioamnionitis, PROM, and EOS rates. In contrast, Apgar's score at 5 minutes and mortality rate were significantly different between groups. In our study, there was a statistically significant difference between groups in terms of day of sampling, this may be due to the earlier screening of the relatively healthy newborns in the control group; in addition, there was no significant difference between the sampling time of confirmed and clinical sepsis groups which consisting mainly of LOS patients.
The mortality rates were $55.9,35.6$, and $12.2 \%$ in the confirmed sepsis, clinical sepsis, and control groups, respectively $(p<0.001)$. Baseline clinical characteristics and demographic data of patients are summarized in - Table 1 .

In the confirmed sepsis group, the blood culture results revealed gram-positive bacteria in 14 patients (23.7\%), gramnegative bacteria in 41 patients (69.5\%), and Candida spp. in four patients (6.8\%). Microbiological findings are shown in - Table 2. The levels of WBC, CRP, and DNI at the onset of sepsis were significantly higher in the sepsis groups than the control group. The sepsis groups showed significantly lower PLT counts. DNI and other laboratory markers of patients are summarized in - Table 3 .

The diagnostic accuracy of markers was examined by ROC curve analysis both individually and in combinations. The optimum cut off for DNI to predict the presence of sepsis was $0.95 \%$ with sensitivity of $60 \%$ and specificity of $94 \%$. The AUCs for the abilities of serum DNI and CRP to predict sepsis (both confirmed and clinical) were 0.84 and 0.95 , respectively (- Table 4). Without presenting a very high AUC, DNI had value comparable to other markers. Furthermore, the combination of DNI and CRP showed the highest sensitivity (86\%), specificity (100\%), and positive predictive value (100\%).

Subgroup analysis was performed in the sepsis groups to assess the association of DNI with sepsis-related mortality. DNI values of nonsurvivors were significantly higher than those of survivors $(p<0.05)$. A cut-off value of DNI at $1.1 \%$ showed sensitivity of $69 \%$, specificity of $77 \%$, positive predictive value

Table 1 Baseline characteristics of patient groups

\begin{tabular}{|l|l|l|l|l|}
\hline & $\begin{array}{l}\text { Confirmed sepsis } \\
\text { group }(\boldsymbol{n}=\mathbf{5 9 )}\end{array}$ & $\begin{array}{l}\text { Clinical sepsis } \\
\text { group }(\boldsymbol{n}=\mathbf{4 6})\end{array}$ & $\begin{array}{l}\text { Control group } \\
(\boldsymbol{n}=\mathbf{4 9})\end{array}$ & $p$-Value \\
\hline Gestational weeks, mean \pm SD & $34 \pm 3$ & $35 \pm 4$ & $34 \pm 3$ & 0.499 \\
\hline Birth weight (g), mean \pm SD & $2,269 \pm 849$ & $2,313 \pm 803$ & $2,251 \pm 790$ & 0.879 \\
\hline Apgar's score at 1 minute, median (min-max) & $7(3-9)$ & $7(3-9)$ & $7(5-9)$ & 0.157 \\
\hline Apgar's score at 5 minutes, median (min-max) & $9(6-10)$ & $8.5(7-10)^{\mathrm{b}}$ & $9(7-10)$ & $\mathbf{0 . 0 1 3}$ \\
\hline Gender, male $n(\%)$ & $27(45.8)$ & $22(47.8)$ & $19(38.8)$ & 0.641 \\
\hline IUGR, $n$ (\%) & $13(22)$ & $11(23.9)$ & $8(16.3)$ & 0.631 \\
\hline Mortality, $n$ (\%) & $33(55.9)^{\mathrm{a}, \mathrm{b}}$ & $16(35.6)^{\mathrm{b}}$ & $6(12.2)$ & $<\mathbf{0 . 0 0 1}$ \\
\hline Mode of delivery, CS, $n(\%)$ & $45(76.3)$ & $34(73.9)$ & $41(83.7)$ & 0.481 \\
\hline Antenatal steroid, $n(\%)$ & $16(27.1)$ & $6(13)$ & $12(24.5)$ & 0.20 \\
\hline Maternal antibiotic usage, $n(\%)$ & $22(37.3)$ & $22(47.8)$ & $14(28.6)$ & 0.15 \\
\hline PROM, $n$ (\%) & $16(27.1)$ & $12(26.1)$ & $7(14.3)$ & 0.23 \\
\hline Chorioamnionitis, $n$ (\%) & $12(20.3)$ & $6(13)$ & $4(8.2)$ & 0.19 \\
\hline EOS, $n$ (\%) & $8(13.6)$ & $10(21.7)$ & & 0.4 \\
\hline Day of sampling, mean \pm SD & $17.2 \pm 13.2^{\mathrm{b}}$ & $13.3 \pm 8.8^{\mathrm{b}}$ & $7.6 \pm 6.9$ & $<\mathbf{0 . 0 0 1}$ \\
\hline
\end{tabular}

Abbreviations: CS, cesarean section; EOS, early onset sepsis; IUGR, intrauterine growth restriction; M, male; PROM, premature rupture of membranes.

Note: Data presented as median (min-max), mean \pm SD, and $n$ (\%). Bold values indicate significant values at the level of $p<0.05$.

aStatistically significant difference compared with clinical sepsis group.

bStatistically significant difference compared with control group. 
Table 2 Causative microorganisms in confirmed sepsis group

\begin{tabular}{|l|l|}
\hline & Total $(\boldsymbol{n}=\mathbf{5 9 )}, \%$ \\
\hline Gram-negative bacteria & \\
\hline Klebsiella pneumonia & $25(42.4)$ \\
\hline Acinetobacter baumannii & $8(13.5)$ \\
\hline Escherichia coli & $3(5)$ \\
\hline Pseudomonas aeruginosa & $2(3.4)$ \\
\hline Serratia marcescens & $1(1.7)$ \\
\hline Stenotrophomonas maltophilia & $1(1.7)$ \\
\hline Klebsiella oxytoca & $1(1.7)$ \\
\hline Gram-positive bacteria & \\
\hline Coagulase negative staphylococcus & $8(13.5)$ \\
\hline Enterococcus faecium & $3(5)$ \\
\hline Enterococcus fecalis & $2(3.4)$ \\
\hline Staphylococcus aureus & $1(1.7)$ \\
\hline Candida spp. & $4(6.8)$ \\
\hline
\end{tabular}

of $62 \%$, and negative predictive value of $82 \%$ for differentiating survivors from nonsurvivors related to sepsis. In logistic regression analysis including birth weight, gestational week, sex, intrauterine growth restriction, blood culture growth, WBC, PLT, CRP, and DNI measurements, and mortality, only the DNI value was significant and mortality was increased by 1.314 times for a $1 \%$ increase in DNI. - Fig. 1 shows the ROC curve for DNI for mortality in the sepsis groups.

\section{Discussion}

The results of this study showed that DNI was significantly higher in the confirmed and clinical sepsis groups compared with the control group. Our study also showed that DNI values were significantly higher in the nonsurvivors than the survivors. DNI represents the IG fraction of the blood, which can be simply calculated from a routine $\mathrm{CBC}$ test. ${ }^{12}$ In the early phases of infection, IGs migrate from the bone marrow into the circulation to participate in host immune defense against bacteria. ${ }^{13}$ As the DNI is an automatically calculated parameter that is independent of operator expertise, it may represent a reliable and useful alternative to manual microscopic count without additional cost and time. A previous study suggested that automated DNI could overcome the limitations of delay and poor accuracy of manual procedures due to the strong correlation between DNI and manual IG count. $^{14}$

Several recent studies have investigated the impact of DNI in the diagnosis of infections and predicting mortality in infected patients. ${ }^{15,16}$ Very limited data are available regarding the usefulness of DNI for the diagnosis of sepsis and prognosis in neonates. The data presented in this study showed significantly higher DNI values in the confirmed and clinical sepsis groups, compared with the control group in neonates. In addition, DNI measurements of neonates in confirmed sepsis group were significantly higher than in patients with clinical sepsis. Similarly, Koh et al investigated the diagnostic significance of DNI and other conventional parameters in neonatal bacteremia, and they showed that DNI increased significantly in the bacteremia group compared with the control group. ${ }^{11}$ Similarly, Lee et al evaluated the usefulness of DNI as a diagnostic factor for neonatal sepsis and reported that mean DNI values were significantly higher in the sepsis group than in controls. In this previous study, the authors also demonstrated significant associations of DNI with positive blood cultures and mortality. ${ }^{10}$ Additionally, Senthilnayagam et al investigated the performance of automated IGs in predicting blood culture results and their clinical utility in 200 febrile patients, and suggested that elevations in automated IG count may be a potential marker

Table 3 Delta neutrophil index and other laboratory markers of patients

\begin{tabular}{|c|c|c|c|c|}
\hline & $\begin{array}{l}\text { Confirmed sepsis } \\
\text { group }(n=59)\end{array}$ & $\begin{array}{l}\text { Clinical sepsis } \\
\text { group }(n=46)\end{array}$ & $\begin{array}{l}\text { Control group } \\
(n=49)\end{array}$ & $p$-Value \\
\hline WBC $\left(10^{3} / \mu \mathrm{L}\right)$ & $14,185 \pm 10,066^{\mathrm{a}}$ & $17,717 \pm 10,968^{b}$ & $12,296 \pm 5,057$ & 0.035 \\
\hline Leukopenia $\left(<5,000 / \mathrm{mm}^{3}\right), n(\%)$ & $9(15.3)$ & $3(6.5)$ & $2(4)$ & \\
\hline Leukocytosis $\left(>20,000 / \mathrm{mm}^{3}\right), n(\%)$ & $15(25.4)$ & $14(30.4)$ & $4(8.2)$ & \\
\hline $\mathrm{PLT}\left(10^{3} / \mu \mathrm{L}\right)$ & $155 \pm 161^{\mathrm{a}, \mathrm{b}}$ & $208 \pm 143^{b}$ & $306 \pm 161$ & $<0.001$ \\
\hline $\begin{array}{l}\text { Thrombocytopenia, } \\
\left(<100 \text { to } \geq 50 / \mathrm{mm}^{3}\right), n(\%)\end{array}$ & $6(10.2)$ & 5 (10.9) & & \\
\hline Thrombocytopenia, $\left(<50 / \mathrm{mm}^{3}\right), n(\%)$ & $28(47.5)$ & $13(28.3)$ & & \\
\hline CRP (mg/L) & $85.6 \pm 84.9^{\mathrm{a}, \mathrm{b}}$ & $49.6 \pm 53.6^{b}$ & $1.9 \pm 2.4$ & $<0.001$ \\
\hline CRP positivity, (>10 mg/L), n (\%) & $48(81.4)$ & $32(69.6)$ & & \\
\hline DNI (\%) & $6.9 \pm 9.3^{a, b}$ & $1.9 \pm 2.1^{b}$ & $0.4 \pm 0.5$ & $<0.001$ \\
\hline
\end{tabular}

Abbreviations: CRP, C-reactive protein; DNI, delta neutrophil index; PLT, platelet; WBC, white blood cell.

Note: Bold values indicate significant values at the level of $p<0.05$.

${ }^{a}$ Statistically significant difference compared with clinical sepsis group.

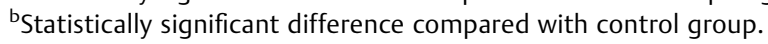


Table 4 Comparison of area under the curve values, sensitivity, specificity, positive predictive value, and negative predictive value of markers both individually and in combinations

\begin{tabular}{|l|l|l|l|l|l|l|l|l|l|}
\hline Test result variable(s) & Area & $p$-Value & Min & Max & Cut-off value & $\begin{array}{l}\text { Sensitivity, } \\
\%\end{array}$ & $\begin{array}{l}\text { Specificity, } \\
\%\end{array}$ & PPV, \% & NPV, \% \\
\hline $\begin{array}{l}\text { WBC } \\
\left(10^{3} / \mu \mathrm{L}\right)\end{array}$ & 0.58 & $\mathbf{0 . 0 4 6}$ & 0.489 & 0.668 & 16,270 & 40 & 82 & 82 & 39 \\
\hline $\begin{array}{l}\mathrm{PLT} \\
\left(10^{3} / \mu \mathrm{L}\right)\end{array}$ & 0.74 & $<\mathbf{0 . 0 0 1}$ & 0.664 & 0.824 & 175 & 61 & 84 & 89 & 50 \\
\hline $\begin{array}{l}\mathrm{CRP} \\
(\mathrm{mg} / \mathrm{L})\end{array}$ & 0.95 & $\mathbf{0 . 0 1 8}$ & 0.914 & 0.983 & 7.45 & 86 & 96 & 98 & 76 \\
\hline $\begin{array}{l}\mathrm{DNI} \\
(\%)\end{array}$ & 0.84 & $\mathbf{0 . 0 3 1}$ & 0.782 & 0.903 & 0.95 & 60 & 94 & 95 & 52 \\
\hline $\mathrm{DNI}+\mathrm{CRP}$ & 0.96 & $<\mathbf{0 . 0 0 1}$ & 0.933 & 0.989 & & 86 & 100 & 100 & 77 \\
\hline $\mathrm{DNI}+\mathrm{PLT}$ & 0.86 & $\mathbf{0 . 0 3 0}$ & 0.800 & 0.916 & & 81 & 84 & 91 & 67 \\
\hline $\mathrm{DNI}+\mathrm{WBC}$ & 0.85 & $\mathbf{0 . 0 3 0}$ & 0.791 & 0.910 & & 77 & 88 & 93 & 64 \\
\hline
\end{tabular}

Abbreviations: CRP, C-reactive protein; DNI, delta neutrophil index; NPV, negative predictive value; PLT, platelet count; PPV, positive predictive value; WBC, white blood cell.

Note: Bold values indicate significant values at the level of $p<0.05$.

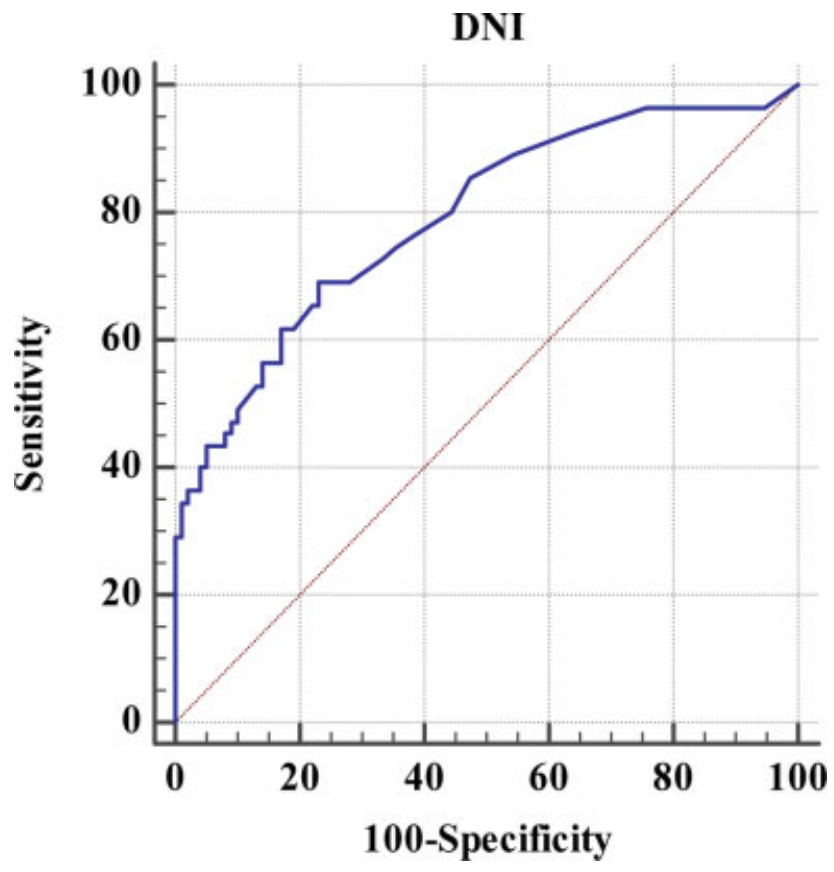

Fig. 1 Receiver operating characteristic curve demonstrating the predictive value of delta neutrophil index for mortality with neonatal sepsis.

for bacteremia, and higher values (IG $>3 \%$ ) showed specificity $>90 \%{ }^{17}$ These findings support our results, which indicate that DNI may be capable of predicting neonatal sepsis.

The results of this study also showed that the DNI had a better correlation with neonatal sepsis than WBC and PLT count, as it had the highest AUC in ROC curves in terms of $\mathrm{CBC}$ parameters. Previously, Hornik et al performed two multicenter cohort studies to evaluate the diagnostic accuracy of $\mathrm{CBC}$ and differentials in both early and late onset neonatal sepsis, and concluded that although low WBC counts and ANC and high immature/total neutrophil ratios are associated with infection, none of these parameters could reliably exclude sepsis in neonates due to their poor sensitivity. ${ }^{18,19}$ Another study in critically ill adult patients with sepsis suggested that DNI was superior to WBC and ANC for discriminating between patients with and without severe sepsis/septic shock. ${ }^{20}$ Therefore, we suggest that $\mathrm{CBC}$ could provide better diagnostic capacity than routinely checked WBC and PLT counts by assessment of DNI.

CRP is one of the most easily available laboratory tests in the diagnosis of neonatal sepsis, but it has several drawbacks. CRP is a late rising biomarker; it takes 10 to 12 hours to obtain a measurable level in the serum. It can also reach a peak level up to 60 hours. ${ }^{21}$ On the other hand, DNI reflects the left shift that appeared at the beginning of the infectious stimulus. In newborn age group which the clinical course can deteriorate rapidly, timely diagnosis is critical to prevent serious complications and mortality. CRP rises several hours after the onset of illness, while the DNI rises 12 hours before the onset of organ/circulatory failure in severe sepsis cases. ${ }^{20}$ DNI can lead to rapid diagnose and faster initiation of treatment with this feature. Further, the half-life of DNI is 3 hours, which is much shorter compared with the 24 to 48 hours of CRP. ${ }^{12,20}$ DNI can lead to rapid diagnose and faster initiation of treatment with these features. In NICUs, it is also aimed to perform less blood sampling to avoid iatrogenic anemia; thus, a painful invasive procedure is reduced too. DNI measurement from $C B C$ which is such a commonly used test, provides less blood sampling, faster results, and no additional costs in the clinical settings. Although DNI alone was less specific than CRP, we thought that it was comparable value due to its advantages. Besides, although the PPV/NPV value of CRP was found high in our study, it is not valid to assess and report its utility in clinical settings according to our results due to its use as one of the diagnostic criteria of neonatal sepsis in this study. In future studies with larger populations, more reliable sensitivity and specificity values with different DNI cutoffs could be revealed. 
In addition, this study also investigated the AUCs for combinations of DNI + WBC, DNI + PLT, and DNI + CRP and revealed that combination of DNI with CRP had the highest AUC with $86 \%$ sensitivity and $100 \%$ specificity for diagnosing sepsis. Similarly, in adults, Seok et al noted that the combination of DNI and CRP more accurately predicted sepsis than either marker alone. ${ }^{22}$ To our knowledge, this is the first study to compare DNI with other conventional infection parameters both individually and in combination for determination of neonatal sepsis. As the sensitivity, specificity, PPV, and NPV ultimately determine the usefulness of diagnostic tests, DNI + CRP would be the best combination for use in this condition. Therefore, in the present study, the interpretation of elevations in both DNI and CRP together would be a more convenient marker than DNI alone at the onset of symptoms.

In this study, at the onset of disease, the median DNI was significantly higher in the nonsurvivor group than in the survivor group with a cut-off value of $1.1 \%$. This was consistent with the results of Kim et al who reported that both DNI at day 1 and DNI trend were significantly associated with early mortality in adult patients with gram-negative bacteremia, with a cut-off value of $7.6 \%{ }^{23}$ Consistent with the present results, Han et al reported that elevated DNI was an independent predictor of mortality in patients with septic acute kidney injury treated with continuous renal replacement therapy. ${ }^{24}$ They also showed that mortality rates were significantly higher in the highest DNI group compared with the intermediate and lowest DNI groups when patients were divided into three groups based on their DNI tertile (high, DNI $>12 \%$; intermediate, $12-3.6 \%$; low, <3.6\%). In contrast, van der Geest et al reported that nonsurvivors did not have a higher automated IGs than survivors among infected patients. ${ }^{25}$ These inconsistencies were likely to be due to the lack of a generally accepted cut-off value, so it is essential to obtain a precise cutoff value to predict mortality for different age groups and medical conditions or even for different automatic analyzers.

This study had several limitations. First, it had a retrospective design and was based on a small population. Second, only one measurement at the onset of disease was included, so the impact of DNI trend on outcomes could not be investigated. Therefore, we were unable to evaluate the response of DNI to empirical antibiotic therapy. Third, in confirmed sepsis group, gram negative microorganisms were dominant, so response of DNI may depends on different bacterial species. In the future, DNI should be further evaluated with extended randomized controlled trials on a larger group of patients to support our hypothesis and overcome our limitations.

In conclusion, this study showed that DNI could be used as a diagnostic marker for neonatal sepsis and may be widely used in clinical practice. The most useful aspect of DNI is that short turnaround time, immediate response to an infectious process, does not requiring additional cost, time, and blood volume in clinical settings. The diagnostic efficiency of the complete blood count in diagnosis of sepsis may be increased by the use of DNI. Furthermore, these advantages become more prominent in neonates with severe medical conditions requiring early intervention, such as sepsis. In addition, the diagnostic capability of DNI may be increased by simultaneous assessment of CRP measurements.

\section{Funding}

None.

Conflict of Interest

None declared.

\section{References}

1 Gilfillan M, Bhandari V. Biomarkers for the diagnosis of neonatal sepsis and necrotizing enterocolitis: clinical practice guidelines. Early Hum Dev 2017;105:25-33

2 van Herk W, Stocker M, van Rossum AM. Recognising early onset neonatal sepsis: an essential step in appropriate antimicrobial use. J Infect 2016;72(72, Suppl):S77-S82

3 Bhandari V. Effective biomarkers for diagnosis of neonatal sepsis. J Pediatric Infect Dis Soc 2014;3(03):234-245

4 Nigro KG, O'Riordan M, Molloy EJ, Walsh MC, Sandhaus LM. Performance of an automated immature granulocyte count as a predictor of neonatal sepsis. Am J Clin Pathol 2005;123(04):618-624

5 Murphy K, Weiner J. Use of leukocyte counts in evaluation of earlyonset neonatal sepsis. Pediatr Infect Dis J 2012;31(01):16-19

6 Cimenti C, Erwa W, Herkner KR, Kasper DC, Müller W, Resch B. The predictive value of immature granulocyte count and immature myeloid information in the diagnosis of neonatal sepsis. Clin Chem Lab Med 2012;50(08):1429-1432

7 Fernandes B, Hamaguchi Y. Automated enumeration of immature granulocytes. Am J Clin Pathol 2007;128(03):454-463

8 Nierhaus A, Klatte S, Linssen J, et al. Revisiting the white blood cell count: immature granulocytes count as a diagnostic marker to discriminate between SIRS and sepsis-a prospective, observational study. BMC Immunol 2013;14:8

9 Sol IS, Park HB, Kim MJ, et al. Delta neutrophil index as a prognostic marker in the pediatric intensive care unit. Korean J Crit Care Med 2016;31(04):351-358

10 Lee SM, Eun HS, Namgung R, Park MS, Park KI, Lee C. Usefulness of the delta neutrophil index for assessing neonatal sepsis. Acta Paediatr 2013;102(01):e13-e16

11 Koh ID, Jeon IS, Kim HM. Diagnostic significance of the delta neutrophil index and other conventional parameters in neonatal bacteremia. Pediatr Infect Vaccine 2017;24(01):1-6

12 Park JH, Byeon HJ, Lee KH, et al. Delta neutrophil index (DNI) as a novel diagnostic and prognostic marker of infection: a systematic review and meta-analysis. Inflamm Res 2017;66(10):863-870

13 Cornbleet PJ. Clinical utility of the band count. Clin Lab Med 2002; 22(01):101-136

$14 \mathrm{Nahm} \mathrm{CH}$, Choi JW, Lee J. Delta neutrophil index in automated immature granulocyte counts for assessing disease severity of patients with sepsis. Ann Clin Lab Sci 2008;38(03):241-246

15 Ahn C, Kim W, Lim TH, Cho Y, Choi KS, Jang BH. The delta neutrophil index (DNI) as a prognostic marker for mortality in adults with sepsis: a systematic review and meta-analysis. Sci Rep 2018;8(01):6621

$16 \operatorname{Lim}$ TS, Kim BK, Lee JW, et al. Use of the delta neutrophil index as a prognostic factor of mortality in patients with spontaneous bacterial peritonitis: implications of a simple and useful marker. PLoS One 2014;9(01):e86884

17 Senthilnayagam B, Kumar T, Sukumaran J, Jeya M, Rao KR. Automated measurement of immature granulocytes: performance characteristics and utility in routine clinical practise. Pathol Res Int 2012;2:483-489

18 Hornik CP, Benjamin DK, Becker KC, et al. Use of the complete blood cell count in late-onset neonatal sepsis. Pediatr Infect Dis J 2012;31(08):803-807 
19 Hornik CP, Benjamin DK, Becker KC, et al. Use of the complete blood cell count in early-onset neonatal sepsis. Pediatr Infect Dis J 2012;31(08):799-802

20 Park BH, Kang YA, Park MS, et al. Delta neutrophil index as an early marker of disease severity in critically ill patients with sepsis. BMC Infect Dis 2011;11:299

21 Odabasi IO, Bulbul A. Neonatal sepsis. Sisli Etfal Hastan Tip Bul 2020;54(02):142-158

22 Seok Y, Choi JR, Kim J, et al. Delta neutrophil index: a promising diagnostic and prognostic marker for sepsis. Shock 2012;37(03): 242-246
$23 \mathrm{Kim} \mathrm{HW}$, Yoon JH, Jin SJ, et al. Delta neutrophil index as a prognostic marker of early mortality in gram negative bacteremia. Infect Chemother 2014;46(02):94-102

24 Han IM, Yoon CY, Shin DH, et al. Delta neutrophil index is an independent predictor of mortality in septic acute kidney injury patients treated with continuous renal replacement therapy. BMC Nephrol 2017;18(01):94

25 van der Geest PJ, Mohseni M, Brouwer R, van der Hoven B, Steyerberg EW, Groeneveld AB. Immature granulocytes predict microbial infection and its adverse sequelae in the intensive care unit. J Crit Care 2014;29(04):523-527 